\title{
Chromosomal Aberrations Associated with Clonal Evolution and Leukemic Transformation in Fanconi Anemia: Clinical and Biological Implications
}

\author{
Stefan Meyer, ${ }^{1,2,3}$ Heidemarie Neitzel, ${ }^{4}$ and Holger Tönnies ${ }^{5}$ \\ ${ }^{1}$ clo Young Oncology Unit, Department of Paediatric and Adolescent Oncology, Christie Hospital, Wilmslow Road, \\ Manchester M20 6XB, UK \\ ${ }^{2}$ Stem Cell \& Leukaemia Proteomics Laboratory, School of Cancer and Enabling Sciences, Faculty of Medical \\ and Human Sciences, University of Manchester, Manchester Academic Health Science Centre, Manchester, UK \\ ${ }^{3}$ Department of Paediatric Oncology, Royal Manchester Children's Hospital Oxford Road, Manchester M13 9WL, UK \\ ${ }^{4}$ Institute for Medical and Human Genetics, Charité Universitätsmedizin Berlin, Germany, Campus Virchow-Klinikum, \\ Augustenburger Platz 1, 13353 Berlin, Germany \\ ${ }^{5}$ Gendiagnostik Kommission, Robert Koch Institut, DGZ-Ring 1, 13086 Berlin, Germany
}

Correspondence should be addressed to Stefan Meyer, stefan.meyer@manchester.ac.uk

Received 6 January 2012; Accepted 13 March 2012

Academic Editor: Laura Hays

Copyright ( 2012 Stefan Meyer et al. This is an open access article distributed under the Creative Commons Attribution License, which permits unrestricted use, distribution, and reproduction in any medium, provided the original work is properly cited.

Fanconi anaemia (FA) is an inherited disease with congenital and developmental abnormalities, bone marrow failure, and extreme risk of leukemic transformation. Bone marrow surveillance is an important part of the clinical management of FA and often reveals cytogenetic aberrations. Here, we review bone marrow findings in FA and discuss the clinical and biological implications of chromosomal aberrations associated with leukemic transformation.

\section{Introduction}

Fanconi anemia (FA) is an inherited disease with bone marrow failure, variable congenital and developmental abnormalities, and extreme cancer predisposition. The most common malignancies in FA are myeloid leukemia and squamous cell carcinoma. On a cellular level, FA is characterized by chromosomal instability and cross-linker sensitivity, which is the diagnostic hallmark of FA. For diagnostic testing, this is determined by demonstration of hypersensitivity to mitomycin C (MMC) or diepoxybutane (DEB) of patient derived peripheral blood cells or fibroblasts [1-3]. FA cells also display hypersensitivity to proapoptotic stimuli of certain cytokines, such as TNF- $\alpha$ and IFN- $\gamma$, which has been implicated in haematological manifestations of FA [4-6]. Cell cycle analysis of FA cells shows a characteristic arrest in the G2 phase, which is exacerbated by exposure to MMC [7-9]. This clinical and cellular phenotype results from a defect in a DNA damage response (DDR) pathway (FA/BRCA pathway), in which FA and associated proteins interact. So far, $15 \mathrm{FA}$ genes (FANCA, FANCB, FANCC, FANCD1/BRCA2, FANCD2, FANCE, FANCF, FANCG, FANCI, FANCJ, FANCL, FANCM, FANCN/PALPB2, FANCO/RAD51C, and FANCP/SLX4) have been identified that can be mutated in FA [2, 10-12], of which FANCA, FANCG, and FANCC are the most commonly mutated genes in studied FA populations [2]. Importantly, the discovery that mutations in BRCA2 causes FA in the subgroup FA-D1, which comprises less than $5 \%$ of all FA patients, linked the FA DNA damage response pathway to hereditary breast and ovarian cancer (HBOC) [13, 14]. Hematopoiesis in the bone marrow (BM) is the most commonly affected organ system in FA, and most FA patients will develop clinically relevant hematological complications in their first or second decades of life [15]. BM complications of FA can manifest with hypoplasia, often initially being limited to thrombocytopenia in peripheral blood counts, or general aplasia. When the diagnosis of FA is made, 
which might happen with considerable delay, bone marrow appearances can already be more advanced and consistent with myelodysplasia. In FA, this often presents as refractory cytopenia with multilineage dysplasia, with or without excess of blasts on morphologic evaluation. Common morphologic abnormalities on bone marrow examination include irregular nuclear contours, budding nuclei, and karyorrhexis [16]. In some patients, the diagnosis of FA is only made on presentation with overt myeloid leukemia. How common undiagnosed FA presents with AML is not known, but FA should be considered especially in young patients with AML, even in the absence of sometimes only subtle congenital malformations such as short stature and microcephaly, in particular when excess toxicity or prolonged aplasia after chemotherapy of extreme toxicity is encountered [17-19]. Less than ten cases of lymphoblastic leukemias have been reported in FA, which have been mostly of T-lineage, and appear to be limited to patients with mutations in FANCD1/BRCA2 and FANCD2 [19-21].

Given the high incidence of hematological complications of FA, BM surveillance for morphological and cytogenetic changes makes an important contribution to the clinical management of FA patients. With improved and more sensitive methods for the detection of chromosomal aberrations over the last decade, and better understanding of clinical implications of BM cytogenetic findings in general for preleukemic changes and the diagnosis and management of hematological malignancies, FA bone marrow surveillance provides important information for clinical decision making. In addition, clonal evolution and associated chromosomal aberrations in FA are important for understanding malignant transformation in general and therefore wider implications. Here, we review bone marrow chromosomal aberrations in FA and discuss the clinical and biological implications.

\subsection{Bone Marrow Surveillance: Clinical Aspects for Patient} Management. In view of the relative infrequency and clinical variety of FA, no evidence-based data exist on how and how frequent bone marrow should be surveyed in FA patients. In view of variable practice, a recent survey carried out in the UK (S. Meyer, unpublished data), confirmed broadly the recommended practice, in that FA patients with normal blood counts should have at least a yearly assessment of bone marrow morphology and cytogenetics $[3,22]$. If there is evidence of bone marrow failure, most centers would consider increasing the frequency and monitor more closely for appearance and evolution of chromosomal aberrations. For the initial management planning at diagnosis, it is worth considering that absent radius and a severe phenotype is statistically associated with earlier bone marrow failure, and that of the most commonly mutated genes, FANCG and FANCC have a statistically higher incidence of early hematological complications than FANCA [23]. However, $\mathrm{BM}$ failure is a common presentation of FA caused by mutations in all complementation groups. Surveillance for FA-associated BM manifestations should include morphology and assessment of cellularity as well as cytogenetic evaluation [3]. The cytogenetic evaluation should include conventional karyotyping. Importantly, however, cytogenetic analysis should specifically include investigations for FAcharacteristic chromosomal aberrations as outlined below. Only with the application of more sophisticated cytogenetic methodologies the incidence and significance of FA-specific aberrations can be determined. These would include routine application of or/and comparative genomic hybridization (CGH) or more sensitive whole genome analysis such as array $\mathrm{CGH}$, in addition to fluorescent in situ hybridization (FISH), targeting FA-specific chromosomal gains and losses on a single cell level.

\subsection{Spectrum of FA-Specific BM Chromosomal Aberrations.} Clonal bone marrow aberrations in individuals affected by FA were first reported as far back as the 1970s and early 1980s, when several studies recognized cytogenetic abnormalities on bone marrow examination of FA patients, many of them noting a high frequency of monosomy 7 , detected by conventional karyotyping $[25,26]$. The clinical observation that the detectability of chromosomal aberrations in bone marrow aspirates of FA patients can vary over time, with clones becoming transiently undetectable, has led to an underestimation of clinical relevance of chromosomal aberrations in FA [27]. In addition, the absence of nonrandom chromosomal rearrangements that are frequently found in AML in particular in childhood, has delayed the recognition and the clinical and prognostic significance of specific aberrations frequently seen in FA [24]. A better understanding of the clinical relevance and biological implications of chromosomal aberrations in FA was achieved over the last decade by analysis of larger case series and the application of modern molecular cytogenetic technologies in addition to conventional karyotyping [16, 28-31]. This has led to the identification and delineation of specific patterns of chromosomal aberrations in FA. In contrast to aberrations seen in sporadic AML in childhood, these are characteristically unbalanced, with gains and losses of chromosomal material during clonal evolution. Frequent for FA are gains of the chromosomal regions $1 \mathrm{q}$ and $3 \mathrm{q}$, as illustrated in Figure 1, and partial or complete loss of chromosome 7 [16, 29-33]. Of these, 3q gains are in particular characteristic of FA. By studying larger numbers of FA patients sequentially, not only the high specificity for FA became evident, but also the clinical implication of 3q gains, of which occurrence indicate transformation to MDS and AML [30,31]. In four independent studies, two from Europe and two from North America, the association of $3 \mathrm{q}$ gains with progression to or presence of FA-related myelodysplasia was confirmed [16, 29-31]. Importantly, gains involving $3 \mathrm{q}$ are only rarely seen in $\mathrm{BM}$ from nonFanconi patients [34-36], while balanced chromosomal aberrations, such as inversions or translocations involving the $3 \mathrm{q}$ are well documented in myeloid malignancies from non-FA patients, in particular in adults $[35,36]$. Therefore, cytogenetic detection of $3 \mathrm{q}$ gains in apparently sporadic cases of MDS or AML would indicate testing for FA. The impact on gene expression resulting from FA-specific gains in the area of common amplification, 3q26-3q29, has only recently been studied and point to an important role of the 

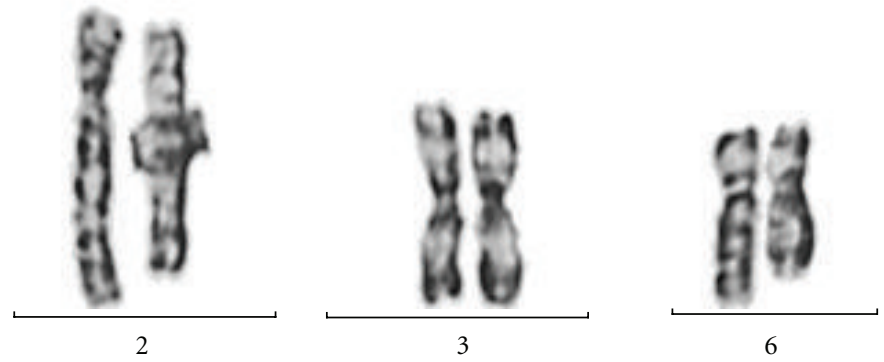

(a)
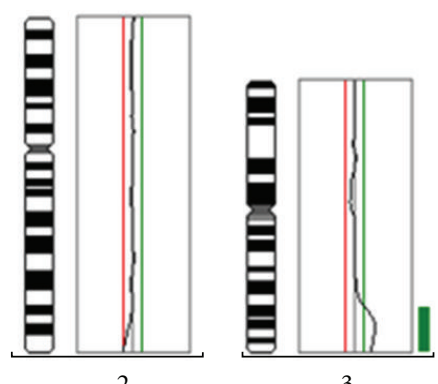

(b)
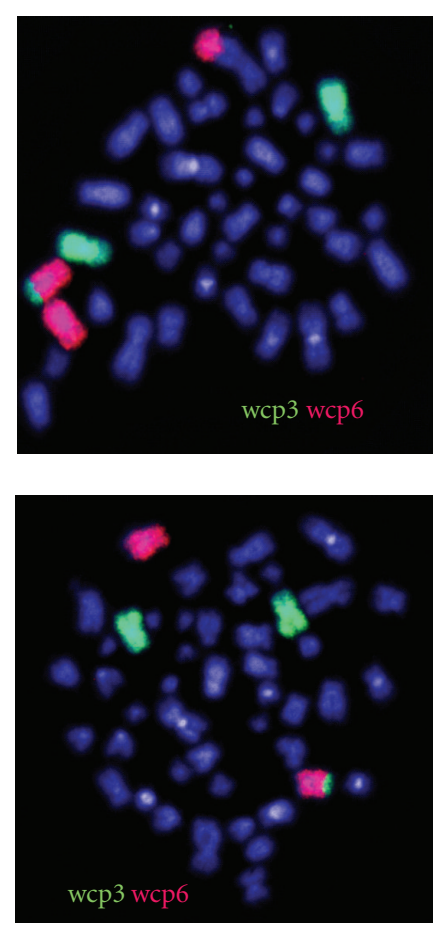

(c)

FIgURE 1: FA-associated 3q aberrations. (a) conventional cytogenetics: outcuts of chromosome 2, 3, and 6 showing additional material at $2 \mathrm{q}$, normal chromosomes 3, and an apparent deletion of $6 \mathrm{q}$. (b) the conventional CGH shows a slight deviation at $2 \mathrm{q}$, a gain of $3 \mathrm{q} 25$ to 3 qter (enh), and a loss of material from $6 \mathrm{q} 23$ to $6 \mathrm{qter}(\mathrm{dim})$. (c) the FISH with whole chromosome paints wcp3 und wcp6 demonstrates two cell lines: one with material of chromosome 6 translocated to $2 \mathrm{q}$ and with material of 3 translocated to $6 \mathrm{q}$; another cell line which carries only the translocation of material of chromosome 3 to $6 \mathrm{q}$. Thus, the apparent deletion detected by conventional cytogenetics proved to be not a sole deletion of $6 \mathrm{q}$ but in addition a unbalanced translocation of $3 \mathrm{q}$ onto $6 \mathrm{q}$. In addition, the patient had a monosomy 7 (data not shown). The karyotype according to ISCN 2009 in bone marrow cells was 45,XY,-7[2]/45, der(6)(6pter $\rightarrow 6 \mathrm{q} 22:: 3 \mathrm{q} 25 \rightarrow 3 \mathrm{qter}),-$ 7[27]/45, der(2)(2pter $\rightarrow$ 2q37::6q22 $\rightarrow$ 6qter), der(6)(6pter $\rightarrow$ 6q22::3q25 $\rightarrow 3 q$ ter $),-7[8]$.

transcriptional regulator EVI1 (ecotropic viral integration site 1) for leukaemic transformation in FA [37, 38]. Another frequently observed aberration in FA is gain of chromosomal material at 1q. This aberration can also be present in morphologically relatively normal BM and is a finding also in non FA-hematological diseases. Its presence is often the sole finding in the early stages of clonal evolution and can persist for years, but also occurs frequently with $3 \mathrm{q}$ gains and other aberrations. Chromosomal aberration involving chromosome 7 include $-7 /-7 \mathrm{q}$, which, as in the non-FA population, is significantly correlated with more advanced dysplasia and commonly part of a clone with a more complex karyotype that frequently also shows gain of $3 \mathrm{q}$ material [39]. Sequential analysis of clonal progression in FA has revealed that $3 \mathrm{q}$-gains often precede changes involving partial or whole loss of chromosome 7 [30, 37]. Another more recently recognized frequent finding in FA-associated clonal evolution is 11q-in advancing FA-associated MDS. This lesion occurs in FA frequently with a more complex karyotype that also shows $3 \mathrm{q}$ gain and/or -7 [31]. The recent detection of involvement of the RUNX1 locus at $21 \mathrm{q}$ in FA-associated genomic abnormalities, which in all cases were associated with advanced MDS [31], has also some important biological implications for the understanding of clonal evolution with FA, which is discussed below. Gains and losses of chromosomal material can also involve other chromosomes, but not with the same FA-specific patterns as for 3q. Balanced translocations have been described in FA, and occur usually as part of more complex clonal rearrangements. Single cases also had involvement of the $3 \mathrm{q}$ region as well a single report of an $11 \mathrm{q} 23$ translocation [21, 38]. Importantly, common balanced nonrandom chromosomal rearrangements that are seen in AML, such as $t(8 ; 21)$ or $\operatorname{inv}(16)$ translocations, have never been reported in FA [24].

\subsection{Clonal FA-Associated Bone Marrow Aberrations: Clinical} Implications. Hematological complications are the most common manifestation of FA. Over the last three decades according to large studies carried out in North America and Europe, the cumulative incidence of any hematological abnormality in FA approaches $90 \%$, and the cumulative incidence of leukemia has been approximately $30 \%$ by 40 years of age $[20,23]$. Therapeutically, options to treat bone marrow failure in FA are limited to interventions with growth factors and androgens in order to improve peripheral blood counts [3], but this does not alter the high risk of leukemic 
transformation. The most important management decision for hematological complications of FA is when and how to proceed to hematopoietic stem cell transplantation (HSCT). The outcome of HSCT in FA has improved dramatically [40, 41 ], and the incidence and survival patterns quoted above, as well the clinical course of FA are changing accordingly [23]. Many centers would elect HSCT for FA in the presence of significant hematological abnormalities and availability of a suitable donor. Cytogenetic information would certainly inform decision making, and the presence of chromosomal aberrations, in particular those associated with high risk of malignant transformation would potentially justify a more aggressive approach that could include partly mismatched donors $[41,42]$. Leukemia in FA is very difficult to treat. From sparse published data of relatively few reported cases, overt leukemia in FA is associated with poor prognosis and short survival. Conditioning regimes for FA have empirically been tailored for the intrinsic chemosensitivity of FA patients and are increasingly based on fludarabine with low-dose cyclophosphamide. There is little evidence that the presence of BM cytogenetic aberrations should influence conditioning regimes, as long as there is no evidence of overt leukemia. However, numbers of reported cases are very small [40, 41, 43], and several cases of leukemic relapse after HSCT have been reported, of which intriguingly one was from donor cells [44].

\subsection{FA-Characteristic Chromosomal Aberrations: Implications} for Malignant Transformation. The emergence of characteristic patterns of chromosomal aberrations in FA has relevance for the management of FA patients. Detection of chromosomal aberrations that confer a high risk of transformation to MDS and AML warrants a more aggressive approach in order to prevent leukemia development. However, the study of chromosomal aberrations in this disorder has some more generally relevant implications, giving insight of secondary events in clonal evolution arising associated with an inherited defect in the DNA damage response. The FA-characteristic clonal evolution with dominance of chromosomal gains and losses is likely to be a specific result of the disruption of the FA/BRCA pathway and at least partially caused by FA-related unresolved DNA damage during $\mathrm{S}$ phase. This unresolved DNA damage is thought to lead to the FA-specific G2 arrest and could lead to the accumulation double-strand breaks and switch to more error-prone repair by nonhomologous end joining. Indeed, nonhomologous end joining is largely efficient in FA cells in contrast to homologous repair, which is grossly impaired in FA [45-47]. However, this possible and extremely simplified explanation could only partly explain the occurrence of aberrations, but not characteristic patterns of chromosomal aberrations involving typically $3 \mathrm{q}$ and $1 \mathrm{q}$. The striking overrepresentation of $1 \mathrm{q}$ and $3 \mathrm{q}$ could imply that these chromosomal regions are particularly susceptible to FA/BRCA disruption-associated damage. An alternative or additional explanation would be that resulting genetic changes confer a growth advantage, possibly in particular in the presence of a defect in the FA/BRCA pathway. Cytogenetic analysis of sporadic AMLs that occur in a comparable age group of older children and young adults shows some marked differences when compared with FAassociated leukaemic transformation, exemplified by the rarity of any $3 q$ aberrations in less than $5 \%$ of childhood the AML in the NPO studies and the MRC trials [48, 49], and exceedingly rare findings of gains of chromosomal material in this region, implying an alternative pathogenesis of FAassociated leukaemic transformation. The FA-characteristic $3 q$ gains nearly always harbor one of the most aggressive leukemogenic oncogenes, EVI1, which was first detected to be amplified and overexpressed as an initial event in FA-derived AML transformation in patient material and cell lines from a patient with biallelic FANCD1/BRCA2 mutations $[16,31,38]$ and subsequently shown to result in overexpression of EVI1 [37]. This suggest that FAassociated leukemia shares its biology with one of the most aggressive forms of sporadic AML $[35,36]$. Intriguingly, EVI1 overexpression in childhood AML, which has been detected in approximately $10 \%$ of cases, is normally not a result of chromosomal rearrangements of the $3 q$ region, but appears to be associated other chromosomal rearrangements and is not of the same prognostic relevance as EVI1 overexpression resulting from chromosomal rearrangements [50]. The other specific gene that appears to be targeted by FA-associated chromosomal rearrangements is RUNX1, which points to the question as to how chromosomal rearrangements in FA promote leukemic transformation. One important observation comes from studies with FANCC -/- mice. Leukaemic clones in bone marrow of these mice that were outgrown under the selective pressure of TNF$\alpha$ showed abrogated cytokine sensitivity occurring together with chromosomal aberrations [51]. In addition, analysis of patient derived BM cells of FA patients with chromosomal aberrations led to the detection of an attenuated cellular FA phenotype. Cells with this phenotype maintained lack of FANCD2 ubiquitination associated with FA core complex gene mutations, and MMC hypersensitivity, but did not display the FA-specific G2 arrest on cell cycle analysis [28]. It will be important to explore to what extent and by which mechanism individual or combined the genetic changes associated with leukemic transformation in FA modulating the cellular FA phenotype. Taken together, these observations point to a modulatory effect mediated by chromosomal aberrations on the cellular FA phenotype, which is likely to be of general relevance for oncogene-mediated malignant progression and the DNA damage response [52].

\section{Acknowledgment}

S. Meyer is supported by Cancer Research UK (CRUK) and Leukaemia Lymphoma Research UK (LLR).

\section{References}

[1] A. D. Auerbach, "Fanconi anemia and its diagnosis," Mutation Research, vol. 668, no. 1-2, pp. 4-10, 2009.

[2] J. P. de Winter and H. Joenje, "The genetic and molecular basis of Fanconi anemia," Mutation Research, vol. 668, no. 1-2, pp. 11-19, 2009. 
[3] A. Shimamura and B. P. Alter, "Pathophysiology and management of inherited bone marrow failure syndromes," Blood Reviews, vol. 24, no. 3, pp. 101-122, 2010.

[4] K. Bijangi-Vishehsaraei, M. R. Saadatzadeh, A. Werne et al., "Enhanced TNF- $\alpha$-induced apoptosis in Fanconi anemia type C-deficient cells is dependent on apoptosis signal-regulating kinase 1," Blood, vol. 106, no. 13, pp. 4124-4130, 2005.

[5] L. S. Haneline, H. E. Broxmeyer, S. Cooper et al., "Multiple inhibitory cytokines induce deregulated progenitor growth and apoptosis in hematopoietic cells from $\mathrm{Fac}(-/-)$ mice," Blood, vol. 91, no. 11, pp. 4092-4098, 1998.

[6] X. Li, Y. Yang, J. Yuan et al., "Continuous in vivo infusion of interferon-gamma (IFN- $\gamma$ ) preferentially reduces myeloid progenitor numbers and enhances engraftment of syngeneic wild-type cells in Fancc-/- mice," Blood, vol. 104, no. 4, pp. 1204-1209, 2004.

[7] B. Dutrillaux, A. Aurias, and A. M. Dutrillaux, "The cell cycle of lymphocytes in Fanconi anemia," Human Genetics, vol. 62, no. 4, pp. 327-332, 1982.

[8] M. C. Heinrich, M. E. Hoatlin, A. J. Zigler et al., "DNA crosslinker-induced G2/M arrest in group C Fanconi anemia lymphoblasts reflects normal checkpoint function," Blood, vol. 91, no. 1, pp. 275-287, 1998.

[9] H. Seyschab, R. Friedl, Y. Sun et al., "Comparative evaluation of diepoxybutane sensitivity and cell cycle blockage in the diagnosis of fanconi anemia," Blood, vol. 85, no. 8, pp. 2233 2237, 1995.

[10] Y. Kim, F. P. Lach, R. Desetty, H. Hanenberg, A. D. Auerbach, and A. Smogorzewska, "Mutations of the SLX4 gene in Fanconi anemia," Nature Genetics, vol. 43, no. 2, pp. 142-146, 2011.

[11] C. Stoepker, K. Hain, B. Schuster et al., "SLX4, a coordinator of structure-specific endonucleases, is mutated in a new Fanconi anemia subtype," Nature Genetics, vol. 43, no. 2, pp. 138-141, 2011.

[12] F. Vaz, H. Hanenberg, B. Schuster et al., "Mutation of the RAD51C gene in a Fanconi anemia-like disorder," Nature Genetics, vol. 42, no. 5, pp. 406-409, 2010.

[13] N. G. Howlett, T. Taniguchi, S. Olson et al., "Biallelic inactivation of BRCA2 in Fanconi anemia," Science, vol. 297, no. 5581, pp. 606-609, 2002.

[14] W. D. Foulkes, "Inherited susceptibility to common cancers," New England Journal of Medicine, vol. 359, no. 20, pp. 20942153, 2008.

[15] A. Butturini, R. P. Gale, P. C. Verlander, B. Adler-Brecher, A. P. Gillio, and A. D. Auerbach, "Hematologic abnormalities in Fanconi anemia: an international Fanconi anemia registry study," Blood, vol. 84, no. 5, pp. 1650-1655, 1994.

[16] A. M. Cioc, J. E. Wagner, M. L. MacMillan, T. DeFor, and B. Hirsch, "Diagnosis of myelodysplastic syndrome among a cohort of 119 patients with fanconi anemia: morphologic and cytogenetic characteristics," American Journal of Clinical Pathology, vol. 133, no. 1, pp. 92-100, 2010.

[17] S. Meyer, L. M. Barber, D. J. White et al., "Spectrum and significance of variants and mutations in the Fanconi anaemia group $G$ gene in children with sporadic acute myeloid leukaemia," British Journal of Haematology, vol. 133, no. 3, pp. 284-292, 2006.

[18] A. Rochowski, P. S. Rosenberg, T. A. Alonzo et al., "Estimation of the prevalence of Fanconi anemia among patients with de novo acute myelogenous leukemia who have poor recovery from chemotherapy," Leukemia Research, vol. 36, no. 1, pp. 2931, 2012.
[19] A. Borriello, A. Locasciulli, A. M. Bianco et al., "A novel Leu153Ser mutation of the Fanconi anemia FANCD2 gene is associated with severe chemotherapy toxicity in a pediatric Tcell acute lymphoblastic leukemia," Leukemia, vol. 21, no. 1, pp. 72-78, 2007.

[20] B. P. Alter, N. Giri, S. A. Savage et al., "Malignancies and survival patterns in the National Cancer Institute inherited bone marrow failure syndromes cohort study," British Journal of Haematology, vol. 150, no. 2, pp. 179-188, 2010.

[21] K. Sugita, T. Taki, Y. Hayashi et al., "MLL-CBP fusion transcript in a therapy-related acute myeloid leukemia with the $\mathrm{t}(11 ; 16)(\mathrm{q} 23 ; \mathrm{p} 13)$ which developed in an acute lymphoblastic leukemia patient with Fanconi anemia," Genes Chromosomes Cancer, vol. 27, no. 3, pp. 264-269, 2000.

[22] A. Shimamura, "Treatment of hematologic abnormalities in Fanconi anemia," in Fanconi Anemia Guidelines for Diagnosis and Management, M. E. Eiler, D. Frohnmayer, L. Frohnmayer, K. Larsen, and J. Owen, Eds., pp. 49-75, 2008.

[23] P. S. Rosenberg, B. P. Alter, and W. Ebell, "Cancer risks in Fanconi anemia: findings from the German Fanconi anemia registry," Haematologica, vol. 93, no. 4, pp. 511-517, 2008.

[24] A. D. Auerbach and R. G. Allen, "Leukemia and preleukemia in Fanconi anemia patients: a review of the literature and report of the International Fanconi anemia registry," Cancer Genetics and Cytogenetics, vol. 51, no. 1, pp. 1-12, 1991.

[25] P. Nowell, G. Bergman, and E. Besa, "Progressive preleukemia with a chromosomally abnormal clone in a kindred with the Estren-Dameshek variant of Fanconi's anemia," Blood, vol. 64, no. 5, pp. 1135-1138, 1984.

[26] V. C. Thurston, T. M. Ceperich, G. H. Vance, and N. A. Heerema, "Detection of monosomy 7 in bone marrow by fluorescence in situ hybridization: a study of Fanconi anemia patients and review of the literature," Cancer Genetics and Cytogenetics, vol. 109, no. 2, pp. 154-160, 1999.

[27] B. P. Alter, A. Scalise, J. McCombs, and V. Najfeld, "Clonal chromosomal abnormalities in Fanconi's anaemia: what do they really mean?" British Journal of Haematology, vol. 85, no. 3, pp. 627-630, 1993.

[28] R. Ceccaldi, D. Briot, J. Larghero et al., "Spontaneous abrogation of the G2 DNA damage checkpoint has clinical benefits but promotes leukemogenesis in Fanconi anemia patients," Journal of Clinical Investigation, vol. 121, no. 1, pp. 184-194, 2011.

[29] P. A. Mehta, R. E. Harris, S. M. Davies et al., "Numerical chromosomal changes and risk of development of myelodysplastic syndrome-acute myeloid leukemia in patients with Fanconi anemia," Cancer Genetics and Cytogenetics, vol. 203, no. 2, pp. 180-186, 2010.

[30] H. Tönnies, S. Huber, J. S. Kühl, A. Gerlach, W. Ebell, and H. Neitzel, "Clonal chromosomal aberrations in bone marrow cells of Fanconi anemia patients: gains of the chromosomal segment 3q26q29 as an adverse risk factor," Blood, vol. 101, no. 10, pp. 3872-3874, 2003.

[31] S. Quentin, W. Cuccuini, R. Ceccaldi et al., "Myelodysplasia and leukemia of fanconi anemia are associated with a specific pattern of genomic abnormalities that includes cryptic RUNX1/AML1 lesions," Blood, vol. 117, no. 15, pp. e161-e170, 2011.

[32] R. Berger, A. Bernheim, and M. Le Coniat, "Chromosomal studies of leukemic and preleukemic Fanconi's anemia patients. Examples of acquired 'chromosomal amplification'” Human Genetics, vol. 56, no. 1, pp. 59-62, 1980. 
[33] R. Berger and P. Jonveaux, "Clonal chromosome abnormalities in Fanconi anemia," Hematology and Cell Therapy, vol. 38, no. 4, pp. 291-296, 1996.

[34] S. Gröschel, S. Lugthart, and R. F. Schlenk, "High EVI1 expression predicts outcome in younger adult patients with acute myeloid leukemia and is associated with distinct cytogenetic abnormalities," Journal of Clinical Oncology, vol. 28, no. 12, pp. 2101-2107, 2011.

[35] S. Lugthart, S. Gröschel, H. B. Beverloo et al., "Clinical, molecular, and prognostic significance of WHO type $\operatorname{inv}(3)(\mathrm{q} 21 \mathrm{q} 26.2) / \mathrm{t}(3 ; 3)(\mathrm{q} 21 ; \mathrm{q} 26.2)$ and various other $3 \mathrm{q}$ abnormalities in acute myeloid leukemia," Journal of Clinical Oncology, vol. 28, no. 24, pp. 3890-3898, 2010.

[36] S. Lugthart, E. Van Drunen, Y. Van Norden et al., "High EVI1 levels predict adverse outcome in acute myeloid leukemia: prevalence of EVI1 overexpression and chromosome 3 q26 abnormalities underestimated," Blood, vol. 111, no. 8, pp. 4329-4337, 2008.

[37] S. Meyer, C. Bristow, M. Wappett et al., "Fanconi anemia (FA)associated $3 \mathrm{q}$ gains in leukemic transformation consistently target EVI1, but do not affect low TERC expression in FA," Blood, vol. 117, no. 22, pp. 6047-6050, 2011.

[38] S. Meyer, W. D. Fergusson, A. D. Whetton et al., "Amplification and translocation of $3 \mathrm{q} 26$ with overexpression of EVII in fanconi anemia-derived childhood acute myeloid leukemia with biallelic FANCDI/BRCA2 disruption," Genes Chromosomes and Cancer, vol. 46, no. 4, pp. 359-372, 2007.

[39] G. Kardos, I. Baumann, S. J. Passmore et al., "Refractory anemia in childhood: a retrospective analysis of 67 patients with particular reference to monosomy 7," Blood, vol. 102, no. 6, pp. 1997-2003, 2003.

[40] M. L. MacMillan and J. E. Wagner, "Haematopoeitic cell transplantation for Fanconi anaemia-when and how?" British Journal of Haematology, vol. 149, no. 1, pp. 14-21, 2010.

[41] J. E. Wagner, M. Eapen, M. L. MacMillan et al., "Unrelated donor bone marrow transplantation for the treatment of Fanconi anemia," Blood, vol. 109, no. 5, pp. 2256-2262, 2007.

[42] S. Chaudhury, A. D. Auerbach, N. A. Kernan et al., "Fludarabine-based cytoreductive regimen and T-cell-depleted grafts from alternative donors for the treatment of high-risk patients with Fanconi anaemia," British Journal of Haematology, vol. 140, no. 6, pp. 644-655, 2008.

[43] P. A. Mehta, T. Ileri, R. E. Harris et al., "Chemotherapy for myeloid malignancy in children with Fanconi anemia," Pediatric Blood and Cancer, vol. 48, no. 7, pp. 668-672, 2007.

[44] B. Gustafsson, J. Moell, K. Leblanc, G. Barbany, S. Soderhall, and J. Winiarski, "Donor cell-derived acute myeloid leukemia after second allogenic cordblood transplantation in a patient with Fanconi anemia," Pediatric Transplantation. In press.

[45] W. Niedzwiedz, G. Mosedale, M. Johnson, C. Y. Ong, P. Pace, and K. J. Patel, "The Fanconi anaemia gene FANCC promotes homologous recombination and error-prone DNA repair," Molecular Cell, vol. 15, no. 4, pp. 607-620, 2004.

[46] V. H. Oestergaard, F. Langevin, H. J. Kuiken et al., "Deubiquitination of FANCD2 Is Required for DNA Crosslink Repair," Molecular Cell, vol. 28, no. 5, pp. 798-809, 2007.

[47] K. J. Patel and H. Joenje, "Fanconi anemia and DNA replication repair," DNA Repair, vol. 6, no. 7, pp. 885-890, 2007.

[48] E. Forestier, S. Heim, E. Blennow et al., "Cytogenetic abnormalities in childhood acute myeloid leukaemia: a Nordic series comprising all children enrolled in the NOPHO-93-AML trial between 1993 and 2001," British Journal of Haematology, vol. 121 , no. 4 , pp. $566-577,2003$.
[49] C. J. Harrison, R. K. Hills, A. V. Moorman et al., "Cytogenetics of childhood acute myeloid leukemia: United Kingdom medical research council treatment trials AML 10 and 12," Journal of Clinical Oncology, vol. 28, no. 16, pp. 2674-2681, 2010.

[50] B. V. Balgobind, S. Lugthart, I. H. Hollink et al., "EVI1 overexpression in distinct subtypes of pediatric acute myeloid leukemia," Leukemia, vol. 24, no. 5, pp. 942-949, 2010.

[51] J. Li, D. P. Sejas, X. Zhang et al., "TNF- $\alpha$ induces leukemic clonal evolution ex vivo in Fanconi anemia group C murine stem cells," Journal of Clinical Investigation, vol. 117, no. 11, pp. 3283-3295, 2007.

[52] J. Bartkova, Z. Hořejší, K. Koed et al., "DNA damage response as a candidate anti-cancer barrier in early human tumorigenesis," Nature, vol. 434, no. 7035, pp. 864-870, 2005. 


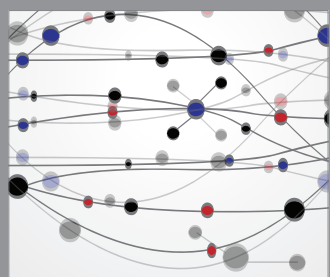

The Scientific World Journal
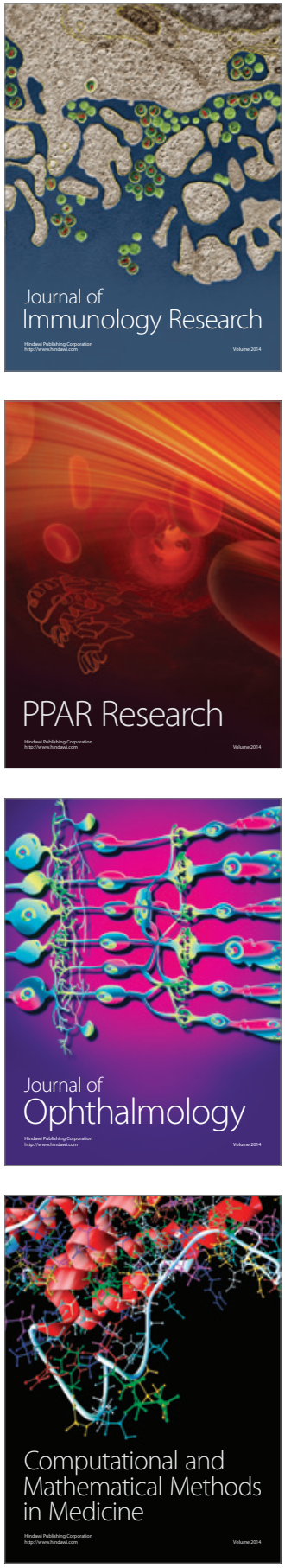

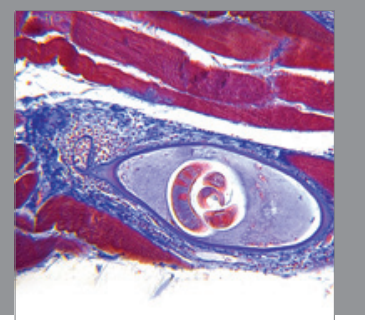

Gastroenterology

Research and Practice
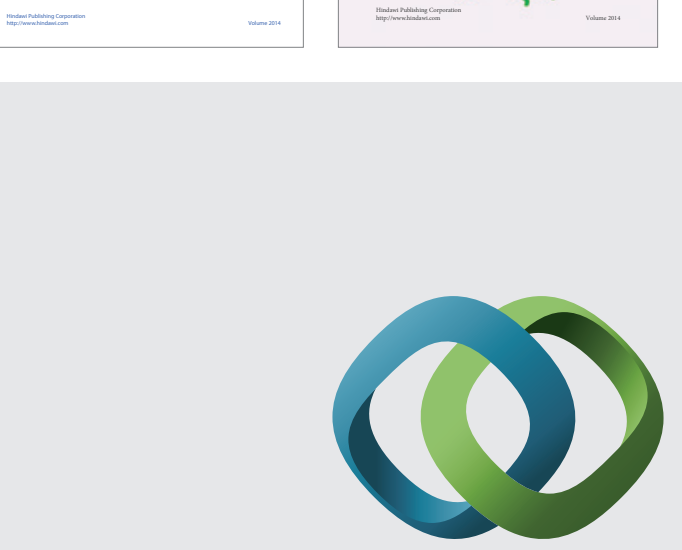

\section{Hindawi}

Submit your manuscripts at

http://www.hindawi.com
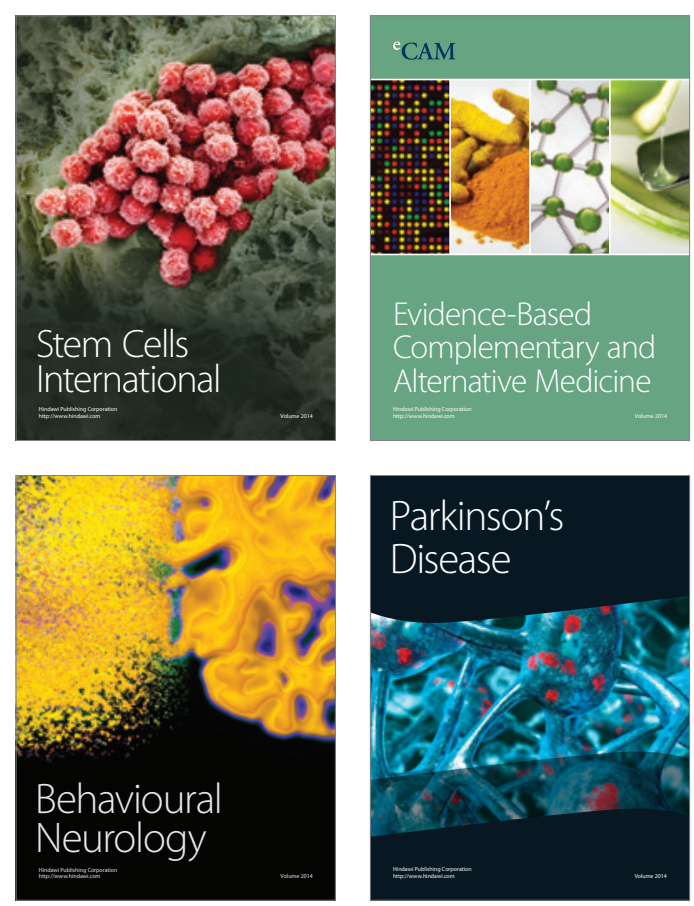

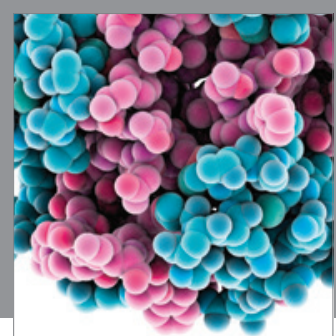

Journal of
Diabetes Research

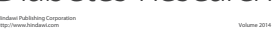

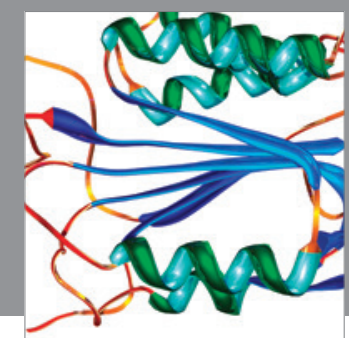

Disease Markers
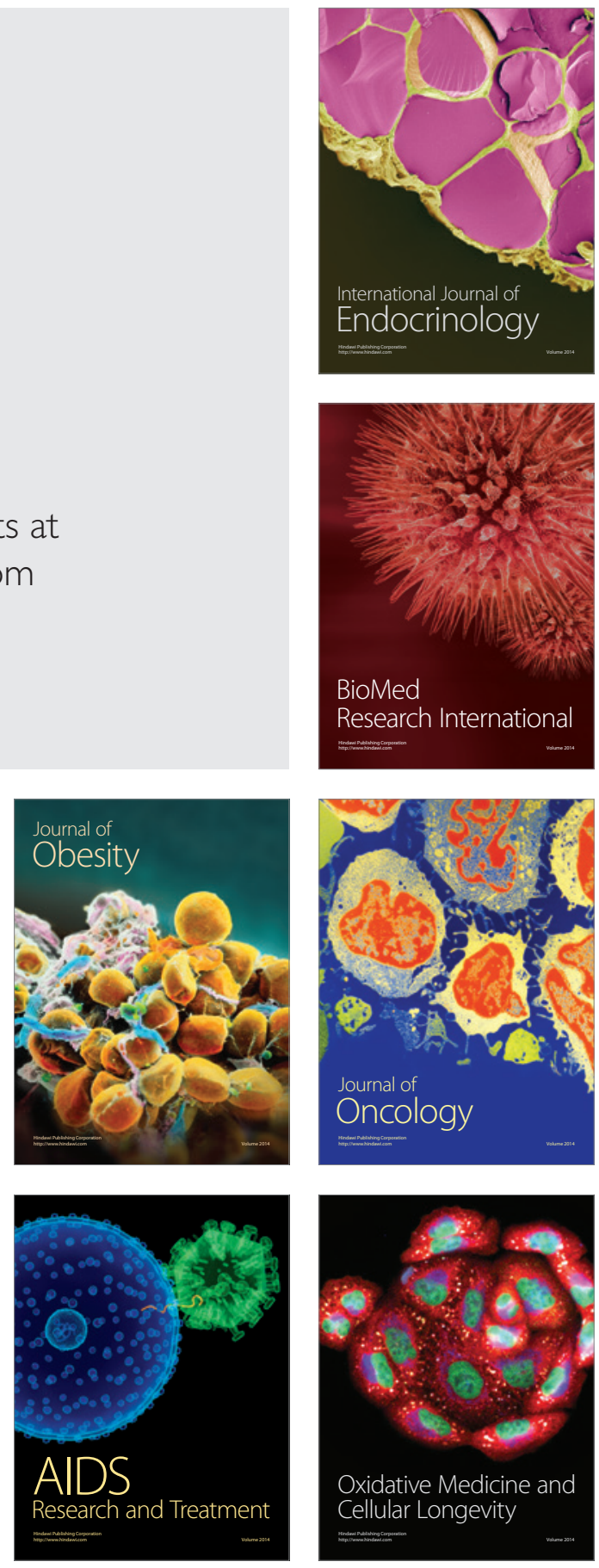